\title{
Laser Pulse Heating of Spherical Metal Particles
}

\author{
Michael I. Tribelsky* \\ Faculty of Physics, M. V. Lomonosov Moscow State University, 1 Lenin Hills, Building 2, Moscow 119991, Russia \\ Moscow State Institute of Radioengineering, Electronics and Automation (Technical University), \\ 78 Vernadskiy Avenue, Moscow 119454, Russia \\ Max-Planck-Institut für Physik komplexer Systeme, Nöthnitzer Str. 38, Dresden 01187, Germany
}

Andrey E. Miroshnichenko and Yuri S. Kivshar

Nonlinear Physics Centre, Research School of Physical Sciences and Engineering, Australian National University, Canberra ACT 0200, Australia

Boris S. Luk'yanchuk ${ }^{\dagger}$

Data Storage Institute, Agency for Science, Technology and Research, Singapore 117608

Alexei R. Khokhlov

\begin{abstract}
A. N. Nesmeyanov Institute of Organoelement Compounds, Russian Academy of Sciences, 28 Vavilova Street, Moscow 119991, Russia Faculty of Physics, M. V. Lomonosov Moscow State University, 1 Lenin Hills, Building 2, Moscow 119991, Russia
\end{abstract}

(Received 1 June 2011; published 27 December 2011)

\begin{abstract}
We consider the general problem of laser pulse heating of spherical metal particles with the sizes ranging from nanometers to millimeters. We employ the exact Mie solution of the diffraction problem and solve the heat-transfer equation to determine the maximum temperature rise at the particle surface as a function of optical and thermometric parameters of the problem. Primary attention is paid to the case when the thermal diffusivity of the particle is much larger than that of the environment, as it is in the case of metal particles in fluids. We show that, in this case, for any given duration of the laser pulse, the maximum temperature rise as a function of the particle size reaches a maximum at a certain finite size of the particle. We suggest simple approximate analytical expressions for this dependence, which cover the entire parameter range of the problem and agree well with direct numerical simulations.
\end{abstract}

DOI: 10.1103/PhysRevX.1.021024

\section{INTRODUCTION}

The problem of laser pulse heating of absorbing particles in transparent media is important for various applications. Initially, the interest in this problem was related to the damage to transparent media initiated by such particles [1]. The area of applications of laser pulse heating has, however, become much broader. The applications may include (but are not limited to) cancer therapy and diagnosis [2,3], microsurgery [4], drug and gene delivery and release $[5,6]$, and nanoscale control of temperature distribution [7]. Local, nanoscale laser heating of magnetic materials beyond the Curie temperature may be employed for superhigh-density data recording and storage [8], etc.

The reason underlying the broad applications of particle heating by laser pulses lies in the fact that a metal nanoparticle excited by a laser pulse with a frequency close to the particle's plasmon resonance can convert the

\footnotetext{
*tribelsky@mirea.ru

†Boris_L@dsi.a-star.edu.sg
}

Published by the American Physical Society under the terms of the Creative Commons Attribution 3.0 License. Further distribution of this work must maintain attribution to the author(s) and the published article's title, journal citation, and DOI.
Subject Areas: Nanophysics, Optics, Plasmonics

electromagnetic energy of the laser pulse into heat efficiently with a dramatic rise of temperature in the surrounding medium. As a result, this general problem (or some parts of it) is the subject of numerous publications in physics, biology, medicine, and chemistry (see, e.g., Refs. [9-13] and references therein).

However, in spite of many experimental studies on this area, theoretical understanding of the phenomenon is rather limited. In a standard approach (see, e.g., a recent paper [14]) the problem is formulated as a study of heat transfer from a source (energy release in the particle owing to absorption of the laser pulse) which is calculated as a solution of the corresponding diffraction problem. Such a problem does not have simple exact analytical solutions, and different studies employ either approximate analytical methods (valid for certain limiting cases only) or direct numerical calculations with the specified parameters. To the best of our knowledge, neither the general solution of this problem, applicable for a broad range of the the parameters of the problem, nor suitable analytical expressions are available in the extensive literature that exists on this subject.

On the other hand, for many applications in physics and other fields, it is highly desirable to obtain the general solution that describes the temperature rise at the surface 
of the particle $T_{s}$ upon the application of a laser pulse in a broad parameter space of the problem, in particular, for particle sizes ranging from nanometers to millimeters. In the present paper we solve this problem, both analytically and numerically.

We demonstrate that the problem can be characterized by four spatial scales, and the competition between them defines the entire variety of different heating regimes. More important, we obtain analytical expressions for the maximum temperature rise at the particle surface as a function of optical and thermometric parameters of the problem. Our results cover a wide range of the particle sizes from a few nanometers to millimeters.

The paper is organized as follows. In Sec. II we formulate the problem and introduce four major spatial scales, namely, the particle size, the thickness of a skin layer, as well as the characteristic lengths of heat diffusion both in the particle and the surrounding medium. These four scales determine a variety of different cases discussed below. Our analytical results and estimates are summarized in Sec. III for twelve different cases, which include all possible combinations of the four spatial scales. Section IV summarizes our numerical results, which are found to be in good agreement with the analytical predictions. Finally, Sec. V concludes the paper.

\section{PROBLEM FORMULATION}

We consider laser heating of a metal particle by a single optical laser pulse with duration $\tau$, see Fig. 1. We assume that the particle is characterized by thermal diffusivity $\chi_{p}$ (typically $\chi_{p} \sim 0.1-1 \mathrm{~cm}^{2} / \mathrm{s}$ ), and it is placed into a fluid with thermal diffusivity $\chi_{f}$ (typically $\chi_{f} \sim$ $10^{-3}-10^{-2} \mathrm{~cm}^{2} / \mathrm{s}$ ). We assume that the condition $\chi_{p} \gg$ $\chi_{f}$ always holds, and that the heat transfer is limited entirely by the heat diffusion. We neglect the influence of convection processes on the temperature profile in the vicinity of the particle. It may be done for the following reasons. First, the characteristic time required for the con-

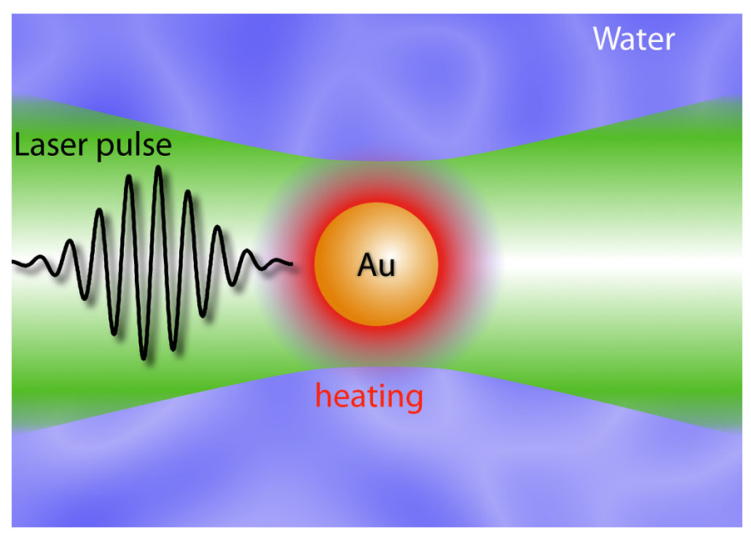

FIG. 1. Schematic of a nanoparticle heated by a laser beam in liquid. vection to arise usually is much larger than the laser pulse duration $\tau$. Second, if the convection eventually arises, the convective flow advects the particle and hence does not affect the temperature profile in its immediate vicinity.

Next, we do not take into account the angular dependence of the heat sources, supposing that the volume density of the sources inside the particle depends just on radial variable $r$. It allows us to replace the actual 3D heattransfer problem by its spherically symmetric version.

The reasons for this approximation are as follows. The actual angular inhomogeneity in the heat sources for small particles is rather weak due to diffractive distortions of the incident light. It results even in weaker temperature inhomogeneities, due to the high rate of heat transfer in metals. Moreover, in what follows, we are interested in estimates of the maximal temperature rise at the particle surface, rather than in its exact calculations. For this reason, we employ the spherically symmetric formulation of the problem even for cases involving large particles, where the illuminated part of a particle under investigation obviously has a temperature higher than the part in shadow.

Under the approximations made, the temperature rise $T$ from the room temperature of the fluid is described by the following standard heat-diffusion problem:

$$
\begin{gathered}
C_{p} \rho_{p} \frac{\partial T}{\partial t}=\kappa_{p} \frac{1}{r^{2}} \frac{\partial}{\partial r}\left(r^{2} \frac{\partial T}{\partial r}\right)+q(r) \quad \text { at } r<R, \\
C_{f} \rho_{f} \frac{\partial T}{\partial t}=\kappa_{f} \frac{1}{r^{2}} \frac{\partial}{\partial r}\left(r^{2} \frac{\partial T}{\partial r}\right) \text { at } r>R, \\
T(0, r)=0, \\
T(t, R-0)=T(t, R+0), \\
\kappa_{p}\left(\frac{\partial T}{\partial r}\right)_{R-0}=\kappa_{f}\left(\frac{\partial T}{\partial r}\right)_{R+0}, \\
T \rightarrow 0, \quad \text { at } r \rightarrow \infty,
\end{gathered}
$$

where $\kappa_{p}$ and $\kappa_{f}$, and $C_{p}$ and $C_{f}, \rho_{p}$, and $\rho_{f}$ stand for the thermal conductivity, specific heat, and density of the particle and environment, respectively, and $q(r)$ is the volume density of heat sources in the particle, which will be specified later. This problem formulation has a certain range of applicability, which should be discussed before we may proceed with an analysis of Eqs. (1)-(6).

First, we suppose that the maximal temperature rise is limited by a few tens of kelvins, so that the fluid always remains below the boiling temperature. It allows us to neglect the temperature dependences of the thermometric parameters of the problem.

Second, in a metal particle electromagnetic energy is absorbed by free electrons and transferred from the electron subsystem to phonons throughout electron-phonon 
collisions. In this case, single joint electron-phonon temperature may be introduced, provided the duration of the laser pulse $\tau$ is larger than the characteristic time of electron-phonon relaxation. At room temperature the frequency of electron-phonon collisions $\nu_{e-\mathrm{ph}}$ in metals is about $10^{13} \mathrm{~s}^{-1}$. For particles whose sizes are larger than the electron mean free path it results in the condition $\tau \gg$ 0.1 ps. For smaller particles, relaxation of the electron subsystem occurs because of collisions of the free electrons with the particle's surface. Consequently, the corresponding condition reads $\tau \gg R / v_{F}$, where $v_{F} \sim 10^{8} \mathrm{~cm} / \mathrm{s}$ stands for the Fermi velocity. For the given values of the constants, the crossover from the condition $\tau \gg 1 / \nu_{e-\mathrm{ph}}$ to $\tau \gg R / v_{F}$ occurs at $R \sim 10^{-5} \mathrm{~cm}$.

Next, we discuss the interface (Kapitza) resistance, which results in a finite temperature jump at the surface of the particle, which may become pronounced at nanoscales [15-17]. The interface resistance brings about the corresponding modifications of the boundary conditions imposed on the particle-fluid interface [14]. Such a modification could be easily incorporated into our model. However, we will not do that for the following reasons: At the room temperature the resistance does not play any role beyond the nanoscale; even at the nanoscale it depends on the wetting properties of the fluid and becomes negligible in the case of good wetting [17]. In the present paper we are interested in the approach rather then in the fine details of a given problem. Therefore, we select the simplest problem formulation, which allows us to demonstrate the approach in the clearest manner. As soon as this task is accomplished, a description of the fine details in most cases can be established in a straightforward matter.

The last issue to be considered concerning the applicability of our theoretical formulation is again related to the size of the particle in question. The macroscopic level of the employed continuum description implies that the particle size should be large relative to the interatomic distance, i.e. $R \gg 10^{-8} \mathrm{~cm}$.

To discuss the laser heating problem defined by Eqs. (1)-(6), we note that the formulation involves the following set of parameters: the intensity (power density in $\mathrm{W} / \mathrm{cm}^{2}$ ) of the laser pulse $I$, its duration $\tau$, frequency $\omega$, and the optical and thermometric constants of the particle and its fluid environment. Following from the linearity of the problem, the heating rate is just proportional to $I$, so the results obtained for a given $I$ may be easily recalculated for any other value by a simple scale transformation; see below. Out of the remaining parameters, four characteristic length scales can be identified: the size of the particle, $R$, which is simply the radius if the particle is spherical, the thickness of the skin layer, $\delta$, which is approximately $\delta \sim 10^{-5} \mathrm{~cm}$ for metals in the optical region, $\sqrt{\chi_{f} \tau}$, and $\sqrt{\chi_{p} \tau}$. According to the general principles of the dimensional analysis, interplay between these four scales determines the entire variety of heating regimes of the particle. Below, all these regimes are discussed and simple approximate analytical solutions for the corresponding temperature rise are obtained.

\section{ANALYTICAL RESULTS}

\section{A. Small particles}

For a small particle we assume that $R \ll \delta$, so that the heating occurs in the entire bulk of the particle rather than on its surface. Interplay between the other scales allows us to consider several different cases, which we analyze below in sequence.

Case S1: $R \ll \delta \ll \sqrt{\chi_{f} \tau} \ll \sqrt{\chi_{p} \tau}$. - In this case the incident light penetrates into the entire particle, and the energy release should be proportional to the particle volume, i.e., the absorption cross section of the particle $\sigma_{\text {abs }} \sim$ $R^{3}$. Bearing in mind that the dimension of $\sigma_{\text {abs }}$ is $\mathrm{cm}^{2}$, it is convenient to write

$$
\sigma_{\mathrm{abs}}=\alpha k R^{3},
$$

where $\alpha$ is a dimensionless quantity and $k=n_{f} \omega / c$ stands for the wave number of the incident light in the fluid. Here $n_{f}$ is the (purely real) refractive index of the fluid and $c$ stands for the speed of light in vacuum.

In the simplest case of the Rayleigh scattering, a comparison of Eq. (7) with the well-known expression for $\sigma_{\mathrm{abs}}$ for a small spherical particle [18] can be employed to obtain the following expression for the coefficient $\alpha$ :

$$
\alpha=\frac{12 \pi \varepsilon^{\prime \prime}}{\left(\varepsilon^{\prime}+2\right)^{2}+\varepsilon^{\prime \prime 2}},
$$

where $\varepsilon^{\prime}$ and $\varepsilon^{\prime \prime}$ stand for the real and imaginary parts of the complex relative dielectric permittivity of the particle $\left(\varepsilon=\varepsilon_{p} / n_{f}^{2}\right)$.

Regarding the energy release caused by dissipation of the incident light, in the case under consideration, a good approximation for it will be a spatially uniform volume density of sources, $q=\sigma_{\text {abs }} I(t) / V$, where $V=(4 / 3) \pi R^{3}$ is the particle volume. The inequality $R \ll \sqrt{\chi_{f} \tau}$ means that the temperature field in the vicinity of the particle is quasisteady (i.e., the term with the temporal derivative of $T$ in the heat-conduction problem may be neglected relative to the terms with spatial derivatives). Therefore, we obtain that within the framework of the approximations made in Eqs. (1) and (2), they are reduced to the following form:

$$
\begin{gathered}
\kappa_{p} \frac{1}{r^{2}} \frac{\partial}{\partial r}\left(r^{2} \frac{\partial T}{\partial r}\right)+\frac{3 \sigma_{\mathrm{abs}} I(t)}{4 \pi R^{3}}=0 \quad \text { at } r<R, \\
\frac{1}{r^{2}} \frac{\partial}{\partial r}\left(r^{2} \frac{\partial T}{\partial r}\right)=0 \quad \text { at } r>R,
\end{gathered}
$$

and $t$ in the dependence $T(r, t)$ plays a role of a parameter.

Integration of Eqs. (8) and (9), with the boundary conditions, Eqs. (4)-(6), yields a parabolic temperature profile at $r<R$ and $T=T_{s}(t) R / r$ at $r>R$. The surface temperature rise of the particle $T_{s}$ may be easily determined 
from the energy balance condition, namely, $\sigma_{\text {abs }} I(t)=$ $-4 \pi R^{2} \kappa_{f} \partial T / \partial r$ at $r=R$, resulting in the following expression:

$$
T_{s}=\frac{\sigma_{\mathrm{abs}} I(t)}{4 \pi R \kappa_{f}} \equiv \frac{\alpha R^{2} k I(t)}{4 \pi \kappa_{f}} .
$$

Note that the temporal dependence of $T_{s}$ coincides with that for $I(t)$, consequently the maximal temperature rise is achieved at the maximum of the profile of the laser pulse.

Case $S 2: R \ll \sqrt{\chi_{f} \tau} \ll \delta \ll \sqrt{\chi_{p} \tau}$. - This case corresponds to a quasisteady temperature field, both in the particle and in the fluid. From the point of view of heat conductivity, the case is identical to S1, therefore, Eq. (10) remains valid.

Case S3: $R \ll \sqrt{\chi_{f} \tau} \ll \sqrt{\chi_{p} \tau} \ll \delta$. -This case is again identical to $S 1$, and Eq. (10) is still applicable.

\section{B. Large particles}

For a large particle, $R \gg \delta$, the energy release occurs in a thin layer near the particle surface.

Case L1: $\delta \ll R \ll \sqrt{\chi_{f} \tau} \ll \sqrt{\chi_{p} \tau}$. - In this case, the absorption cross section should be proportional to the square of the linear size of the particle, i.e.,

$$
\sigma_{\mathrm{abs}}=\pi R^{2} Q_{\mathrm{abs}},
$$

where $Q_{\mathrm{abs}}$ stands for the dimensionless efficiency.

The temperature field remains quasisteady under the condition, $R \ll \sqrt{\chi_{f} \tau} \ll \sqrt{\chi_{p} \tau}$. The difference between the previous case and this one is that now the heat sources can be well approximated by the isotropic energy release at the surface of the particle. Then, the temperature field inside the particle should satisfy the homogeneous Laplace equation. The only nonsingular solution of this equation is a constant profile, in other words, $T(r, t)$ at $r<R$ is reduced to $T(t)$, where time $t$ once again is regarded as a parameter. The temperature field in the fluid environment keeps the same profile as that in cases where $R \ll \delta$. Combining this fact and Eq. (11), we have the following expression for $T_{s}$ :

$$
T_{s}=\frac{\sigma_{\mathrm{abs}} I(t)}{4 \pi R \kappa_{f}} \equiv \frac{Q_{\mathrm{abs}} R I(t)}{4 \kappa_{f}} .
$$

Case L2: $\delta \ll \sqrt{\chi_{f} \tau} \ll R \ll \sqrt{\chi_{p} \tau}$. - In this case, the temperature field inside the particle is still quasisteady, therefore its $r$ dependence may be neglected, similar to case L1 discussed above. The field outside the particle, however, depends on both $t$ and $r$. To determine $T_{s}$ we may employ the energy-conservation law. For simplicity, we consider a rectangular laser pulse with intensity $I_{0}$ and duration $\tau$. Over a certain moment of time $t \leq \tau$, the energy $W$ absorbed by the particle is $\sigma_{\text {abs }} I_{0} t$.

The absorbed energy $W$ is consumed to heat the particle (leading to a rise in $T_{s}$ ) and to heat an adjacent layer of the fluid. The former requires energy of $(4 / 3) \pi R^{3} C_{p} \rho_{p} T_{s}$, the latter $4 \pi R^{2} 2 \sqrt{\chi_{f} t} C_{f} \rho_{f} T_{s} / 2$ (to enhance accuracy of the estimate we have taken into account that the scale of a layer heated over time $t$ is $2 \sqrt{\chi_{f} t}$ [19] and have replaced the profile of the temperature rise in the heated layer by its mean value $T_{s} / 2$ ).

Equating $W$ to the total consumed energy and considering the equality as an equation for unknown $T_{s}$, one easily derives

$$
\begin{aligned}
T_{s}(t) & =\frac{\sigma_{\mathrm{abs}} I_{0} t}{\frac{4}{3} \pi R^{3} C_{p} \rho_{p}+4 \pi R^{2} C_{f} \rho_{f} \sqrt{\chi_{f}} t} \\
& \equiv \frac{Q_{\mathrm{abs}} I_{0} t}{\frac{4}{3} R C_{p} \rho_{p}+4 C_{f} \rho_{f} \sqrt{\chi_{f}}} .
\end{aligned}
$$

The obtained $T_{s}(t)$ is a monotonic function of $t$, therefore, the maximal temperature rise is achieved at the end of the laser pulse. Replacing $t$ by $\tau$ leads to the corresponding expression for the maximal temperature rise $\max _{0<t<\tau} T_{s}$ during the laser pulse $\tau$.

Case L3: $\delta \ll \sqrt{\chi_{f} \tau} \ll \sqrt{\chi_{p} \tau} \ll R$.-According to the employed problem formulation, this case corresponds to the heating of an infinite compound space whose left semispace has the thermometric properties of the particle and whose right one has those of the fluid, and the energy is released at the boundary between the semispaces. This problem is exactly solvable [19]. The solution yields the following expression for the surface-temperature rise:

$$
\begin{aligned}
T_{s}(t) & =\frac{\sigma_{\mathrm{abs}} I_{0}}{2 R^{2} \pi \sqrt{\pi}} \frac{\sqrt{\chi_{p} \chi_{f} t}}{\kappa_{p} \sqrt{\chi_{f}}+\kappa_{f} \sqrt{\chi_{p}}} \\
& \equiv \frac{\alpha I_{0}}{2 \pi \sqrt{\pi}} \frac{\sqrt{\chi_{p} \chi_{f} t}}{\kappa_{p} \sqrt{\chi_{f}}+\kappa_{f} \sqrt{\chi_{p}}} .
\end{aligned}
$$

$T_{s}$ is a monotonic function of time, so the maximal temperature rise is achieved in the end of the pulse, at $t=\tau$.

Case L4: $\sqrt{\chi_{f} \tau} \ll \delta \ll R \ll \sqrt{\chi_{p} \tau}$. - This case is identical to L2; it follows that Eq. (13) is valid.

Case L5: $\sqrt{\chi_{f} \tau} \ll \delta \ll \sqrt{\chi_{p} \tau} \ll R$.- The case is identical to L3, and Eq. (14) is valid.

\section{Other cases}

Case O1: $\sqrt{\chi_{f} \tau} \ll R \ll \delta \ll \sqrt{\chi_{p} \tau}$.- -From the viewpoint of the heat transfer, this case is equivalent to L2, but the absorption cross section is described by Eq. (7). As a result,

$$
\begin{aligned}
T_{s}(t) & =\frac{\sigma_{\mathrm{abs}} I_{0} t}{\frac{4}{3} \pi R^{3} C_{p} \rho_{p}+4 \pi R^{2} C_{f} \rho_{f} \sqrt{\chi_{f}} t} \\
& \equiv \frac{\alpha k I_{0} t}{\frac{4}{3} \pi C_{p} \rho_{p}+4 \pi C_{f} \rho_{f} \frac{\sqrt{\chi_{f} t}}{R}} .
\end{aligned}
$$

Case $02: \sqrt{\chi_{f} \tau} \ll R \ll \sqrt{\chi_{p} \tau} \ll \delta$. This case is identical to O1, and Eq. (15) is valid. 
TABLE I. Summary of qualitatively different regimes of the laser heating, for small and large metal particles in fluids.

\begin{tabular}{|c|c|c|c|}
\hline \multicolumn{2}{|c|}{ Small particles, $R \ll \delta$} & \multicolumn{2}{|c|}{ Large particles, $R \gg \delta$} \\
\hline Condition & Surface temperature, $T_{s}$ & Condition & Surface temperature, $T_{s}$ \\
\hline \multirow{5}{*}{$R \ll \sqrt{\chi_{f} \tau}$} & \multirow{5}{*}{$\frac{\alpha R^{2} k I(t)}{4 \pi \kappa_{f}}$} & \multicolumn{2}{|l|}{ Long pulses } \\
\hline & & $R \ll \sqrt{\chi_{f} \tau} \ll \sqrt{\chi_{p} \tau}$ & $\frac{Q_{\mathrm{abs}} R I(t)}{4 \kappa_{f}}$ \\
\hline & & \multirow{2}{*}{$\sqrt{\chi_{f} \tau} \ll R \ll \sqrt{\chi_{p} \tau}$} & $Q_{\mathrm{abs}} I_{0} t$ \\
\hline & & & $\frac{4}{3} R C_{p} \rho_{p}+4 C_{f} \rho_{f} \sqrt{\chi_{f} t}$ \\
\hline & & $\delta \ll \sqrt{\chi_{p} \tau} \ll R$ & $\frac{\alpha I_{0}}{2 \pi \sqrt{\pi}} \frac{\sqrt{\chi_{p} \chi_{f} t}}{p \sqrt{\chi_{f}}+\kappa_{f} \sqrt{\chi_{p}}}$ \\
\hline \multirow{3}{*}{$\sqrt{\chi_{f} \tau} \ll R$} & & \multicolumn{2}{|l|}{ Short pulses } \\
\hline & $\alpha k I_{0} t$ & \multirow{2}{*}{$\sqrt{\chi_{p} \tau} \ll \delta \ll R$} & $Q_{\mathrm{abs}} I_{0} t$ \\
\hline & $\overline{\frac{4}{3}} \pi C_{p} \rho_{p}+4 \pi C_{f} \rho_{f} \frac{\sqrt{\chi_{f} t}}{R}$ & & $\overline{4\left(C_{p} \rho_{p} \delta+C_{f} \rho_{f} \sqrt{\chi_{f} t}\right)}$ \\
\hline
\end{tabular}

Case O3: $\sqrt{\chi_{f} \tau} \ll \sqrt{\chi_{p} \tau} \ll R \ll \delta$.-According to the approximation used, the energy is released inside the particle in a spatially uniform manner. This case is analogous to O1, and again, Eq. (15) is valid.

Case 04: $\sqrt{\chi_{f} \tau} \ll \sqrt{\chi_{p} \tau} \ll \delta \ll R$.-In this case, the energy release occurs in a surface layer with the volume $4 \pi R^{2} \delta$. The condition $\sqrt{\chi_{p} \tau} \ll \delta$ allows us to neglect the distortion of the temperature inside the layer by heat conductivity. The released energy is consumed to heat the mentioned layer and a layer of the fluid with thickness $2 \sqrt{\chi_{f} t}$. The energy balance yields the following expression for $T_{s}$ :

$$
\begin{aligned}
T_{s}(t) & =\frac{\sigma_{\mathrm{abs}} I_{0} t}{4 \pi R^{2}\left(C_{p} \rho_{p} \delta+C_{f} \rho_{f} \sqrt{\chi_{f} t}\right)} \\
& \equiv \frac{Q_{\mathrm{abs}} I_{0} t}{4\left(C_{p} \rho_{p} \delta+C_{f} \rho_{f} \sqrt{\chi_{f} t}\right)}
\end{aligned}
$$

The maximal temperature rise is achieved at the end of the laser pulse action, at $t=\tau$.

Thus, depending on the relative sizes of the four spatial scales, we can obtain a number of approximate analytical results. By use of inequality $\chi_{f} \ll \chi_{p}$, all these results may be presented as shown in Table I. To understand Table I we should clarify that if a parameter is not explicitly specified in a cell of the table, its value may be any of those permitted for the corresponding column. For example, condition $R \ll \sqrt{\chi_{f} \tau}$ in column, Small particles, $R \ll \delta$ means any of the following three cases: $R \ll \delta \ll$ $\sqrt{\chi_{f} \tau}, \quad R \ll \sqrt{\chi_{f} \tau} \ll \delta \ll \sqrt{\chi_{p} \tau}$, and $R \ll \sqrt{\chi_{f} \tau} \ll$ $\sqrt{\chi_{p} \tau} \ll \delta$. Other cells should be understood in a similar manner.

\section{NUMERICAL VERSUS ANALYTICAL RESULTS}

The accuracy of the developed approach is inspected by a comparison of the obtained analytical expressions with direct numerical simulations of the corresponding spherically symmetric version of the heat-diffusion equation for a gold particle in water; see Eqs. (1)-(6). At $R \leq \delta$ the volume density of heat sources $q$ is taken as spatially uniform within the entire volume of the particle, i.e., $q=$ $3 \sigma_{\text {abs }} I(t) /\left(4 \pi R^{3}\right)$. At $R>\delta$ it is regarded as spatially uniform within the spherical layer with thickness $\delta$, whose upper boundary coincides with the surface of the particle: $q=\sigma_{\text {abs }} I(t) / V$ inside the layer and 0 elsewhere. Here $V$ stands for the volume of the specified spherical layer. At the simulations, $\sigma_{\text {abs }}$ is taken from the exact Mie solution [20] for the gold particle in water. The explicit CrankNickolson method with an adaptive mesh is employed. The particle is heated by a rectangular laser pulse. For definiteness, the wave number of the laser beam (in vacuum) and its intensity are assigned the following typical values: $\lambda=$ $532 \mathrm{~nm}$ (the second harmonic of a Nd:YAG laser) and $I_{0}=$ $5 \times 10^{4} \mathrm{~W} / \mathrm{cm}^{2}$. Regarding the pulse duration $\tau$, its value is different in different runs, which allows us to study various heating regimes (see below). The values of thermometric constants for the particle (gold) and the fluid (water) used in calculations are the following: $\kappa_{p}=318 \frac{\mathrm{W}}{\mathrm{mK}}, \rho_{p}=18.9 \times 10^{3} \frac{\mathrm{kg}}{\mathrm{m}^{3}}, C_{p}=130 \frac{\mathrm{J}}{\mathrm{kg} \mathrm{K}}, \kappa_{f}=$ $0.58 \frac{\mathrm{W}}{\mathrm{mK}}, \rho_{p}=950 \frac{\mathrm{kg}}{\mathrm{m}^{3}}$, and $C_{f}=4200 \frac{\mathrm{J}}{\mathrm{kg} \mathrm{K}}$.

At the wavelength of the incident light, the refractive index of gold is as follows: $n_{p}=n_{p}^{\prime}+i n_{p}^{\prime \prime}=0.45+2.40 i$ [21]. The corresponding value of $\delta$ is associated with the decay of the field of the Poynting vector and equals to $c /\left(2 \omega n_{p}^{\prime \prime}\right)$. At the given wavelength it yields $\delta \approx 17 \mathrm{~nm}$. The purely real refractive index of water is assigned the value $n_{f}=1.33$. In some cases, values of $R$ and/or $\tau$ are extended beyond the range of applicability of the developed approach; see Secs. II and III. We do it deliberately to be able to compare the numerical and analytical results in certain points crucial for the problem in question, such as, e.g., transition points from one heating regime to another. 
To discuss the results of the simulations, we first note that the dependence of the dimensionless quantities $\alpha$ and $Q_{\text {abs }}$ on $R$ usually is very weak except for a narrow region centered about $R \sim \delta$, where the law $\sigma_{\text {abs }} \sim R^{3}$ is replaced by $\sigma_{\text {abs }} \sim R^{2}$. To illustrate this point, we present the obtained dependencies $\sigma_{\mathrm{abs}}(R)$ and the corresponding $Q_{\text {abs }}(R)$ in Fig. 2.

The cubic dependence $\sigma_{\mathrm{abs}}(R)$ (linear dependence of $Q_{\mathrm{abs}}$, i.e., independence of $\alpha$ of $R$ ) at $R<\delta$ is seen clearly. After the passage of the region of sharp variations at $R \sim \delta$, the efficiency $Q_{\text {abs }}$ becomes $R$ independent at any further increase in $R$, i.e., our assumption $\sigma_{\text {abs }} \sim R^{2}$ for large particles does capture the main $R$ dependence of $\sigma_{\text {abs }}$.

It means that the main dependence of $T_{s}$ on the particle size is given by the explicit $R$ dependence of Eqs. (10) and (12)-(16). It is seen straightforwardly in Figs. 3-5, where the dependence of the maximum of the surfacetemperature rise over the duration of the laser pulse $\left(\max _{0<t<\tau} T_{s}\right)$ is plotted as a function of $R$ at various (indicated in the figure captions) values of $\tau$, which represent different heating regimes [22]. For example, as depicted in Fig. 3, an increase in $R$ with other parameters of the problem held fixed, which corresponds to the sequential change of cases S1 and L1-L3, first results in a quadratic growth of $\max _{0<t<\tau} T_{s}$ (case $\mathrm{S} 1$ ), which then is replaced by approximately a linear growth at $R \sim \delta$ (case L1). Next, $\max _{0<t<\tau} T_{s}$ reaches its absolute maximum at $R \sim \sqrt{\chi_{f} \tau}$, declines with a further increase in $R$ (case L2), and finally approaches a constant value at $R \gg \sqrt{\chi_{p} \tau}$ (case L3). For other cases, the dependence of $\max _{0<t<\tau} T_{s}$ on radius $R$ is treated in a similar manner. It should be stressed that the overall comparison of the obtained analytical results with the direct numerical simu-

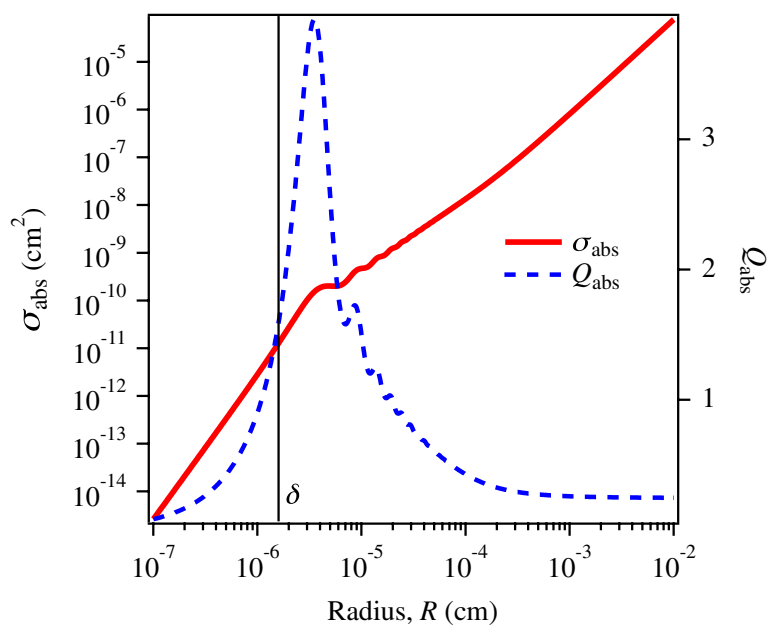

FIG. 2. Absorption cross section $\sigma_{\text {abs }}$ and the corresponding dimensionless efficiency $Q_{\text {abs }}$ for a spherical gold particle in water at $\lambda=532 \mathrm{~nm}$ (in vacuum) and $\delta(\lambda) \approx 17 \mathrm{~nm}$ as functions of the particle radius $R$. Calculations were performed according to the exact Mie solution and experimental data for optical properties of gold [21].

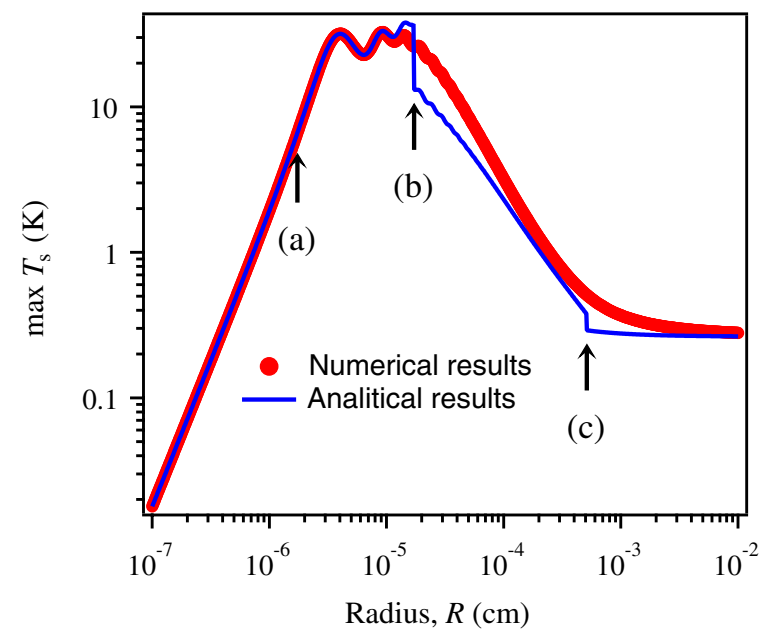

FIG. 3. Comparison of the analytical and numerical results for the maximal temperature rise $\max _{0<t<\tau} T_{s}$ at the surface of a spherical gold particle with radius $R$ in water heated by a rectangular laser pulse with the wavelength, $532 \mathrm{~nm}$ (in vacuum), $I_{0}=5 \times 10^{4} \mathrm{~W} / \mathrm{cm}^{2}, \tau=50 \mathrm{~ns}$. The points of transitions from one heating regime to another are indicated by black arrows: (a) $R=\delta$, (b) $R=2 \sqrt{\chi_{f} \tau}$, and (c) $R=2 \sqrt{\chi_{p} \tau}\left(\delta \ll \sqrt{\chi_{f} \tau}\right.$; see cases S1 and L1-L3).

lation for the entire variety of the heating regimes presented in Figs. 3-5 indicates high accuracy of these results.

Note that there are two competing mechanisms underlying the appearance of the maximum in the surface-temperature rise. The first is related to optics, being associated with the local maximum of $Q_{\text {abs }}$ at $R \simeq \delta$; see Fig. 2 . The second is related to the heat-transfer problem. It is associated with the change of the quasisteady, $R$-dependent temperature field in

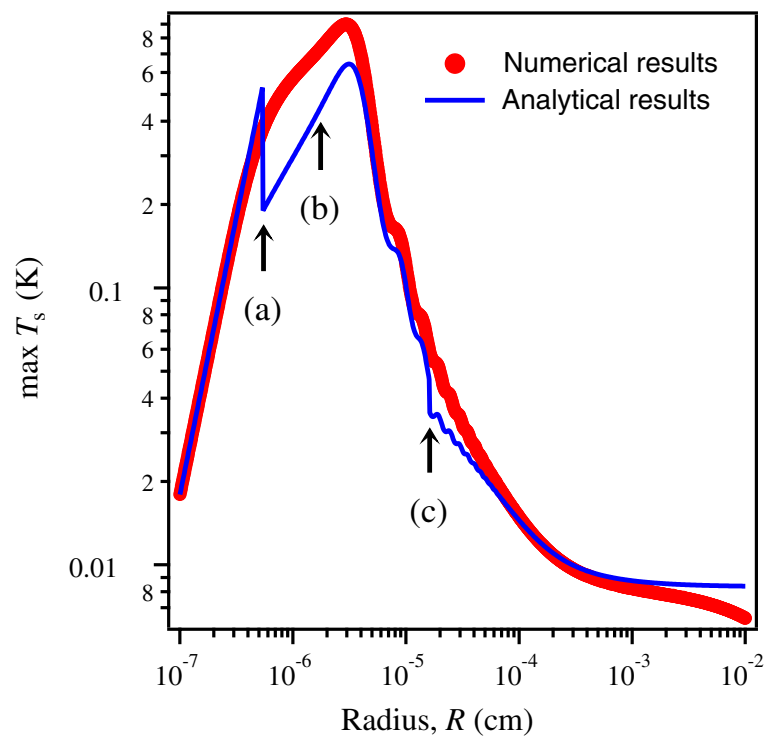

FIG. 4. The same as that shown in Fig. 3 at $\tau=50$ ps. The points of transitions from one heating regime to another are indicated by black arrows: (a) $R=2 \sqrt{\chi_{f} \tau}$, (b) $R=\delta$, and (c) $R=2 \sqrt{\chi_{p} \tau}$ $\left(\sqrt{\chi_{f} \tau} \ll \delta \ll \sqrt{\chi_{p} \tau}\right.$; see cases S2, O1, and L4-L5). 


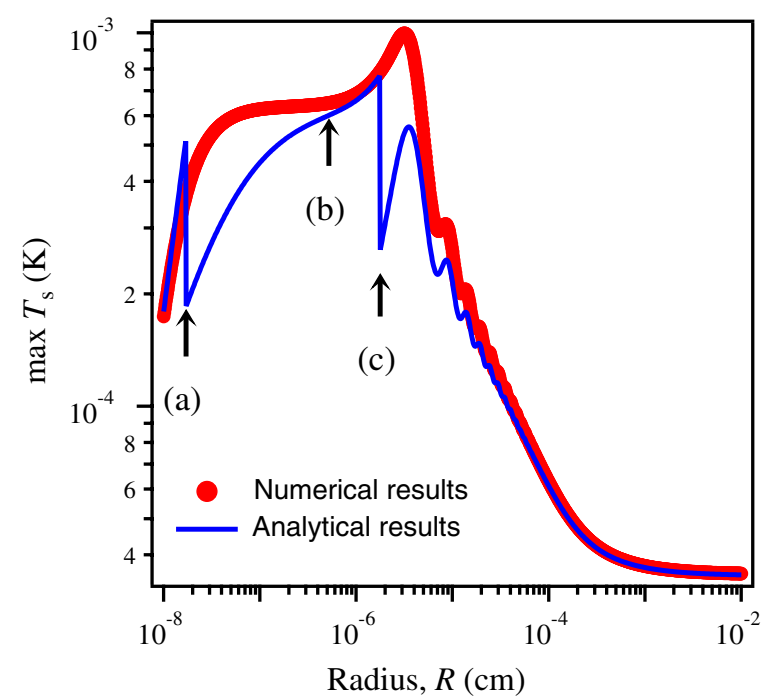

FIG. 5. The same as that shown in Fig. 3 at $\tau=50$ fs. The points of transitions from one heating regime to another are indicated by black arrows: (a) $R=2 \sqrt{\chi_{f} \tau}$, (b) $R=2 \sqrt{\chi_{p} \tau}$, and (c) $R=\delta\left(\sqrt{\chi_{p} \tau} \ll \delta\right.$; see cases $\mathrm{S} 3$, and $\left.\mathrm{O} 2-\mathrm{O} 4\right)$.

the vicinity of the particle to the essentially time-dependent temperature profile, which does not depend on $R$. The change occurs at $R \approx 2 \sqrt{\chi_{f} \tau}$, which singles out this value of $R$ as a second characteristic scale in the $T_{s}$ maximization problem.

To understand the relative role of these mechanisms we plot the maximum of the surface-temperature rise over the duration of the pulse $\max _{0<t<\tau} T_{s}$ as a function of $R$ and $\tau$; see Fig. 6. At every given $R, \max _{0<t<\tau} T_{s}$ increases monotonically with an increase in $\tau$ until the latter reaches the values $\tau \simeq R^{2} /\left(4 \chi_{f}\right)$. Then, the maximal

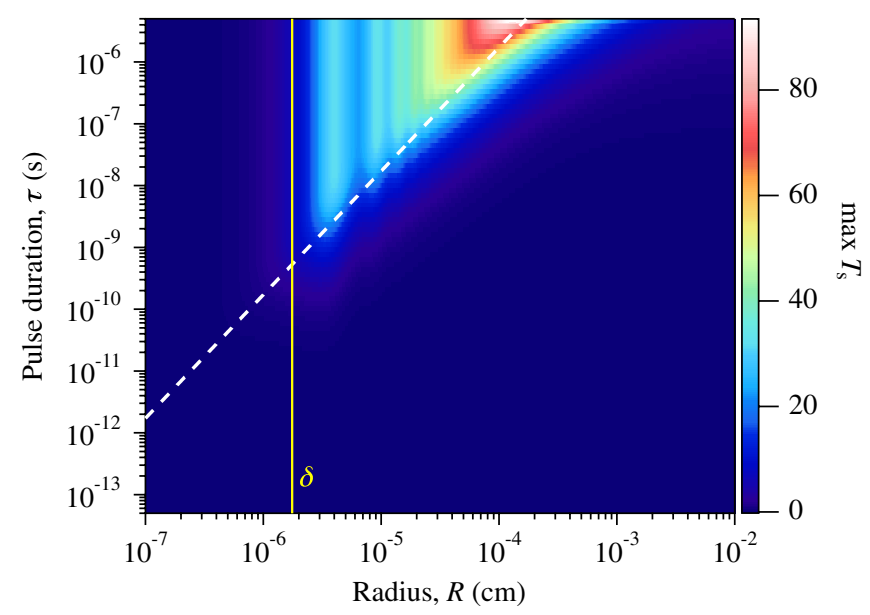

FIG. 6. Density plot of the maximum of the surfacetemperature rise $\max _{0<t<\tau} T_{s}$ for a gold particle in water irradiated by a rectangular laser pulse with $I_{0}=5 \times 10^{4} \mathrm{~W} / \mathrm{cm}^{2}$ and the wavelength, $532 \mathrm{~nm}$ (in vacuum), as a function of the duration of the pulse $\tau$ and the particle radius $R$. The dashed line corresponds to $R=2 \sqrt{\chi_{f} \tau}$. Above this line the temperature rise becomes $\tau$ independent; see Eqs. (10) and (12). surface-temperature rise becomes independent of the duration of the pulse.

Next, we perform the following calculations. For every given $\tau$ we find such a value of $R=R_{\max }$ that maximizes $\max _{0<t<\tau} T_{s}$ and introduce a new quantity $T_{s}^{\mathrm{Max}}=\max _{R} \max _{0<t<\tau} T_{s}$, corresponding to the absolute maximum of the surface-temperature rise, which may be achieved for the given $\tau$ and any value of $R$. Then, $R_{\max }$ and $T_{s}^{\mathrm{Max}}$ are plotted as functions of $\tau$; see Fig. 7. It is easy to see that, for the problem in question, heating for several degrees and higher begins at $\tau \geq 10^{-9} \mathrm{~s}$, when the heattransfer mechanism of the temperature rise maximization problem prevails over the optic one. Let us stress that, as it has been already pointed out in Sec. II, as a consequence of the linearity of the problem, the temperature rise is just proportional to $I_{0}$. This linear relationship allows us to recalculate easily the results obtained for a given value of $I_{0}$ to any other value.

To illustrate how our findings may be employed in various applications, let us consider an important example of selective laser photo-thermal therapy for cancer [23]. In that case, gold nanoparticles of $40 \mathrm{~nm}$ in size that were conjugated to certain antibodies and then incubated with both human oral cancer cells and nonmalignant skin cells were preferentially and specifically bound to the cancer cells. Next, the nanoparticle-labeled cells were exposed to a $\mathrm{cw}$ argon-ion laser at $514 \mathrm{~nm}$. It was found that the malignant cells required less than half the laser energy to be killed as compared to the benign cells. The destruction of the cancer cells occurred owing to laser heating of the gold nanoparticles up to a certain threshold temperature.

On the other hand, in applying this approach in vivo to avoid unwanted effects, one should minimize the exposure

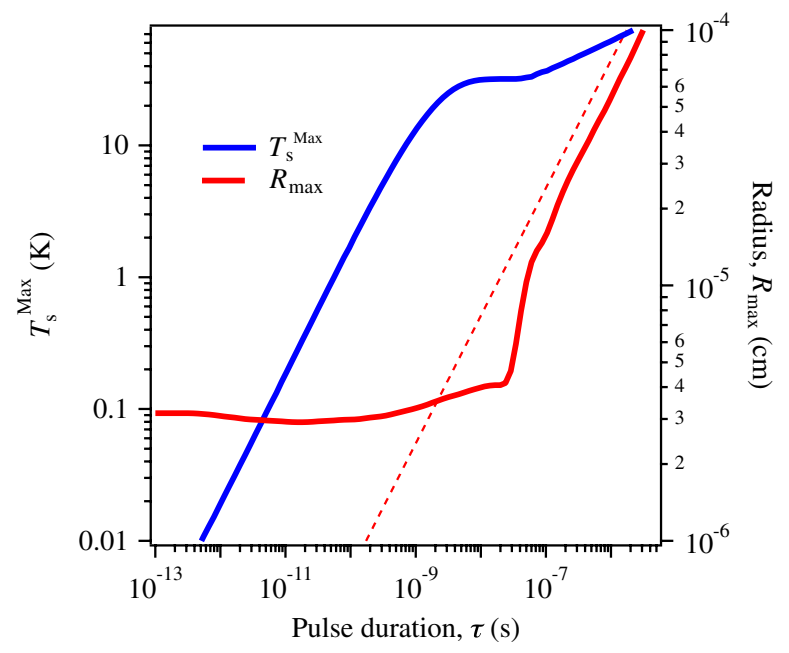

FIG. 7. The radius $R_{\max }$ providing the absolute maximum of the surface-temperature rise and the corresponding temperature rise $T_{s}^{\mathrm{Max}}$ as functions of the laser pulse duration $\tau$ for a gold particle in water irradiated by a rectangular laser pulse with $I_{0}=$ $5 \times 10^{4} \mathrm{~W} / \mathrm{cm}^{2}$ and the wavelength, $532 \mathrm{~nm}$ (in vacuum). The dashed line indicates the dependence $R=2 \sqrt{\chi_{f} \tau}$. 
to the laser beam of the benign tissue and even the tumor itself, maximizing the rate of energy delivered to the nanoparticles and stored in the particles and their immediate vicinity. To this end, a pulse laser should be employed. According to the results presented in Fig. 6 for a particle with a $20 \mathrm{~nm}$ radius, the maximal surface-temperature rise saturates at $\tau>1 \mathrm{~ns}$. Thus, reduction of the pulse duration from infinity (for a $\mathrm{cw}$ laser) to a nanosecond scale (without change of $I_{0}$ ) does not affect $\max _{0<t<\tau} T_{s}$. Presumably, the heating of the nanoparticles by such a pulse still should be fatal for the cancer cells bound to them, while the exposure of the rest of the tumor and the benign tissues will be reduced dramatically.

\section{CONCLUSION}

We have shown that the rather complex problem of laser pulse heating of metal particles in transparent fluids may be described by relatively simple analytical expressions. Our results determine the maximum temperature rise at the particle surface, $T_{s}(t)$, as a function of the particle size and other parameters of the problem. We have demonstrated, by employing direct numerical simulations, that the obtained analytical expressions remain valid and attain a high accuracy for any practically important size of the particle and duration of the laser pulse. More important, at fixed values of the material constants, the maximal surfacetemperature rise $\max _{0<t<\tau} T_{s}$ reaches local maxima at $R \approx \delta$ and $R \approx 2 \sqrt{\chi_{f} \tau}$. For a gold particle in water, considerable heating occurs at $\tau \geq 10^{-9} \mathrm{~s}$, when the absolute maximum of temperature is achieved at $R \approx 2 \sqrt{\chi_{f} \tau}$. We believe that these results, in addition to providing of the most general solution to the problem of laser pulse heating of metal particles, may also be useful for optimizing the efficiency of laser pulse heating of nanoparticles in various problems of laser-matter interactions.

\section{ACKNOWLEDGMENTS}

The work was partially supported by the Australian Research Council, A*STAR projects on metamaterials, and the TSRP Program, Grant No. 102152 0018. M. T. thanks the Max-Planck-Institut für Physik komplexer Systeme for their kind hospitality and support during the substantial part of this project.

[1] A. A. Manenkov and A. M. Prokhorov, Laser-Induced Damage in Solids, Sov. Phys. Usp. 29, 104 (1986).

[2] X. Huang, P. K. Jain, I. H. El-Sayed, and M. A. El-Sayed, Plasmonic Photothermal Therapy (PPTT) Using Gold Nanoparticles, Lasers Med. Sci. 23, 217 (2008).
[3] I. Brigger, C. Dubernet, and P. Couvreur, Nanoparticles in Cancer Therapy and Diagnosis, Adv. Drug Delivery Rev. 54, 631 (2002).

[4] R. R. Anderson and J.A. Parrish, Selective Photothermolysis: Precise Microsurgery by Selective Absorption of Pulsed Radiation, Science 220, 524 (1983).

[5] G. Han, P. Ghosh, M. De, and V.M. Rotello, Drug and Gene Delivery Using Gold Nanoparticles, NanoBiotechnology 3, 40 (2007).

[6] A. G. Skirtach, C. Dejugnat, D. Braun, A. S. Susha, A. L. Rogach, W. J. Parak, H. Möhwald, and G. B. Sukhorukov, The Role of Metal Nanoparticles in Remote Release of Encapsulated Materials, Nano Lett. 5, 1371 (2005).

[7] G. Baffou, R. Quidant, and F. J. García de Abajo, Nanoscale Control of Optical Heating in Complex Plasmonic Systems, ACS Nano 4, 709 (2010).

[8] L. Pan and D.B. Bogy, Heat-Assisted Magnetic Recording, Nat. Photon. 3, 189 (2009).

[9] A. N. Volkov, C. Sevilla, and L. V. Zhigilei, Numerical Modeling of Short Pulse Laser Interaction with Au Nanoparticle Surrounded by Water, Appl. Surf. Sci. 253, 6394 (2007).

[10] H. H. Richardson, M. T. Carlson, P.J. Tandler, P. Hernandez, and A.O. Govorov, Experimental and Theoretical Studies of Light-to-Heat Conversion and Collective Heating Effects in Metal Nanoparticle Solutions, Nano Lett. 9, 1139 (2009).

[11] G. W. Hanson and S. K. Patch, Optimum Electromagnetic Heating of Nanoparticle Thermal Contrast Agents at RF Frequencies, J. Appl. Phys. 106, 054309 (2009).

[12] E. Sassaroni, K.C.P. Li, and B.E. O'Neill, Numerical Investigation of Heating of a Gold Nanoparticle and the Surrounding Microenvironment by Nanosecond Laser Pulses for Nanomedicine Applications, Phys. Med. Biol. 54, 5541 (2009).

[13] S. Bruzzone and M. Malvaldi, Local Field Effects on Laser-Induced Heating of Metal Nanoparticles, J. Phys. Chem. 113, 15805 (2009).

[14] G. Baffou and H. Rigneault, Femtosecond-Pulsed Optical Heating of Gold Nanoparticles, Phys. Rev. B 84, 035415 (2011).

[15] O. M. Wilson, X. Hu, D. G. Cahill, and P. V. Braun, Colloidal Metal Particles as Probes of Nanoscale Thermal Transport in Fluids, Phys. Rev. B 66, 224301 (2002).

[16] Z. Ge, D. G. Cahill, and P. V. Braun, AuPd Metal Nanoparticles as Probes of Nanoscale Thermal Transport in Aqueous Solution, J. Phys. Chem. B 108, 18870 (2004).

[17] L. Xue, P. Keblinski, S. R. Phillpot, S. U.-S. Choi, and J. A. Eastman, Two regimes of Thermal Resistance at a LiquidSolid Interface, J. Chem. Phys. 118, 337 (2003).

[18] L.D. Landau, L. P. Pitaevskii, and E. M. Lifshitz, Electrodynamics of Continuous Media (ButterworthHeinemann, Oxford, 2002).

[19] H.S. Carslow and J.C. Jaeger, Conduction of Heat in Solids (Oxford Univeristy Press, Oxford, 1959).

[20] M. Born and E. Wolf Principles of Optics (Cambridge University Press, Cambridge, 1999).

[21] E. D. Palik, Handbook of Optical Constants of Solids (Academic Press, Orlando, 1985). 
[22] To fill gaps between the regimes, the strong inequalities in their applicability conditions $(\ll)$ are replaced by weak ones $(\leq)$. Thus, cases S1 and L1 in Fig. 3 are matched at $R=\delta$, etc. It explains the discontinuities observed in Figs. 3-5 in the matching points of the different regimes in plots representing the analytical solutions.
[23] I. H. El-Sayed, X. Huang, and M. A. El-Sayed, Selective Laser Photo-Thermal Therapy of Epithelial Carcinoma Using Anti-EGFR Antibody Conjugated Gold Nanoparticles, Cancer Letters (Shannon, Ireland) 239, 129 (2006). 